\title{
Combustion Waves from a Sequential Exothermic and Endothermic Reaction
}

\author{
$\underline{\text { C. Qian }}^{\text {a }}$, H. S. Sidhu ${ }^{\text {a }}$, J. J. Sharples ${ }^{\text {a }}$, I.N. Towers ${ }^{\text {a }}$, V. V. Gubernov ${ }^{\mathrm{b}}$ \\ ${ }^{\mathrm{a}}$ Applied and Industrial Mathematics Research Group, School of Physical, Environmental and \\ Mathematical Sciences, University of New South Wales, Canberra, Northcott Drive, Canberra ACT, \\ 2600, Australia \\ ${ }^{\mathrm{b}}$ I.E. Tamm Theory Department, P.N. Lebedev Physical Institute of Russian Academy of Science, \\ Moscow 119991, RUSSIAN FEDERATION \\ Email: chao.qian@student.adfa.edu.au
}

\begin{abstract}
We consider a one-dimensional flame propagation in a two-stage reaction of a solid fuel under adiabatic conditions. The first reaction is assumed to be exothermic, whereas the second reaction is endothermic. The speed of the front is estimated numerically using a commercial finite element package and was independently verified using the Method of Lines. When the ratio of the heat released by the exothermic to the endothermic reactions is reduced, the significance of the endothermic reaction increases, and the structure of the flame front changes. In particular, the temperature profile begin to develop a more pronounced local maximum close to the flame front as the endothermicity effects are increased. This is unlike the one-step reactions, or even competitive reactions, where such local maxima do not occur. Numerically, we were only able to obtain solutions up to a particular value of this exothermic/endothermic ratio, below which the numerical solutions obtained had difficulties converging to a reliable solution. As our numerical investigation shows there are parameter values in which the flame front no longer travels with a constant speed, but exhibits oscillatory behaviour in the speed. These pulsating regimes in flame propagation has been observed numerically in one-step, and other multi-step reaction schemes. Furthermore, experiments dating as far back as in the early 1950s have recorded such pulsating behaviour. However, these were regarded as unusual behaviour and very little explanation was given to their origins.
\end{abstract}

Keywords: two-stage reactions, exothermic, endothermic, combustion wave, flame speed 


\section{INTRODUCTION}

Combustion is a fundamental process which is important in a wide range of applications, including industrial and environmental process modelling. In simple terms, combustion entails the exothermic reaction between a fuel substance and oxygen to produce combustion products and heat. A number of authors have studied combustion in this context, treating the combustion process as a single-step reaction (Weber et al. (1997), Mclntosh et al. (2004)). In reality, however, the precise chemistry is often far more complicated, involving a number of intermediate reaction steps, some of which are possibly endothermic. The rates of the various reactions involved ultimately determine the energy released, which causes changes in the surrounding temperature. These temperature changes then have a feedback effect on the reaction rates so that the entire process comprises a complex interaction between the chemical species involved, the temperature and the reaction rates.

In recognition of this, a number of authors have analysed so-called multi-step reaction schemes (see Dold (2007) and Liu et al. (1998) all references quoted therein). Typically, these analyses involve only two or three reaction steps, which are chosen to represent the most significant or rate-limiting reaction steps without comprimising the analytical amenability of the resulting systems. For example, two-step, chainbranching combustion schemes were studied by (Dold (2007), Gubernov et al. (2006) etc), two- and threestep parallel exothermic-endothermic reaction schemes (e.g. quenching problems) were studied by Simon et al. (2005) and Gubernov et al. (2008), two-step competitive exothermic-endothermic reaction schemes were studied by Hmaidi et al. (2010) and Sharples et al. (2011), and a two-step sequential combustion scheme was studied by Please et al. (2003). In particular, the latter authors considered the problem of direct reduction of iron ore in iron ore/coal composites and were thus lead to consider the case where one reaction was exothemic and one was endothermic. The motivation of these authors' investigation was from a Mathematics-In-Industry Study Group in 1994 for BHP Research (Hewitt (1995)). We will refer to such a scheme as a sequential exothermic-endothermic combustion scheme. In the present paper we extend the work of Please et al. (2003) by analysing the two-step sequential exothermic-endothermic combustion schemes under more general circumstances.

Specifically we consider the following reaction scheme:

$$
Y_{1} \rightarrow Y_{2}+Q_{1} \rightarrow P+Q_{2}
$$

Here the first reaction proceeds at the temperature-dependent rate $k_{1}(T)$, transforming the first reactant $Y_{1}$ into the intermediate product $Y_{2}$ and heat $Q_{1}$. The second reaction proceeds at a rate $k_{2}(T)$ and transforms the intermediate species $Y_{2}$ into the final product $P$ and heat $Q_{2}$. In the exothermic-endothermic scheme considered it is assumed that $Q_{1}>0$ (exothermic) and $Q_{2}<0$ (endothermic).

The work of Please et al. (2003) only considered condensed phase combustion in the large activation energy limit. By contrast our investigation consider the combustion scheme over a wide range of possible activation energies. In particular, we provide a detailed analysis of the endothermic effects on combustion wave speeds and the propagating temperature profiles. We also demonstrate the existence of oscillatory unstable combustion waves.

\section{GOVERNING EQUATION}

Following Please et al. (2003), we consider a one-dimensional combustion scheme under adiabatic conditions. Assuming the fuel is uniform, premixed and the rate of both reactions are well-described by the Arrhenius law, we obtain the governing partial differential equations (PDEs) as:

$$
\begin{aligned}
\frac{\partial y_{1}}{\partial t} & =-k_{1} y_{1} e^{-E_{1} / R T} \\
\frac{\partial y_{2}}{\partial t} & =-k_{2} y_{2} e^{-E_{2} / R T}+k_{1} y_{1} e^{-E_{1} / R T} \\
\rho c_{p} \frac{\partial T}{\partial t} & =K \frac{\partial^{2} T}{\partial x^{2}}+\rho Q_{1} k_{1} y_{1} e^{-E_{1} / R T}+\rho Q_{2} k_{2} y_{2} e^{-E_{2} / R T}
\end{aligned}
$$


where $x$ and $t$ are the spatial and time coordinates, $T$ is the temperature, and $y_{1}$ and $y_{2}$ are the normalised concentrations of first and second reactants. The other terms are defined as follows:

$\rho$ - density of the fuel $\left(\mathrm{kg} \mathrm{m}^{-3}\right)$

$c_{p}$ - specific heat of the fuel $\left(\mathrm{J} \mathrm{kg}^{-1} \mathrm{~K}^{-1}\right)$

$K$ - thermal conductivity of fuel $\left(\mathrm{J} \mathrm{s}^{-1} \mathrm{~m}^{-1} \mathrm{~K}^{-1}\right)$

$Q_{1}$ - heat of first reaction $\left(\mathrm{J} \mathrm{kg}^{-1}\right)$

$Q_{2}$ - heat of second reaction $\left(\mathrm{J} \mathrm{kg}^{-1}\right)$

$k_{1}$ - pre-exponential rate constant of first reactant $\left(\mathrm{s}^{-1}\right)$

$k_{2}$ - pre-exponential rate constant of second reactant $\left(\mathrm{s}^{-1}\right)$

$E_{1}$ - activation energy of first reaction $\left(\mathrm{J} \mathrm{mol}^{-1}\right)$

$E_{2}$ - activation energy of second reaction $\left(\mathrm{J} \mathrm{mol}^{-1}\right)$

$R$ - universal gas constant $\left(8.314 \mathrm{~J} \mathrm{~mol}^{-1} \mathrm{~K}^{-1}\right)$

For the initial state the two reactants may both be present. Thus, we set $y_{1}=a$ and $y_{2}=1-a$ with $0 \leq a \leq 1$ representing the proportion of fuel at the initial state. For the unburnt region, $x \rightarrow-\infty$, we have $T=0, y_{1}=a$. For the burnt region, $x \rightarrow \infty$, we have $\frac{\partial T}{\partial t}=0, y_{1}=0$.

Defining the non-dimensional temperature, and space and time coordinates as:

$$
u=R T / E_{2}, \quad \xi=x \sqrt{\frac{\rho\left|Q_{2}\right| k_{2} R}{K E_{2}}}, \quad \tau=\left(\frac{\left|Q_{2}\right| k_{2} R}{E_{2} c_{p}}\right) t
$$

the problem can then be written in the non-dimensional form:

$$
\begin{aligned}
\frac{\partial y_{1}}{\partial \tau} & =-\alpha_{2} \beta y_{1} e^{-\alpha_{3} / u} \\
\frac{\partial y_{2}}{\partial t} & =-\beta y_{2} e^{-1 / u}+\alpha_{2} \beta y_{1} e^{-\alpha_{3} / u} \\
\frac{\partial u}{\partial \tau} & =\frac{\partial^{2} u}{\partial \xi^{2}}+\alpha_{1} \alpha_{2} y_{1} e^{-\alpha_{3} / u}+\alpha_{0} y_{2} e^{-1 / u}
\end{aligned}
$$

where the new parameters are

$$
\alpha_{0}=\frac{Q_{2}}{\left|Q_{2}\right|}, \quad \alpha_{1}=\frac{Q_{1}}{\left|Q_{2}\right|}, \quad \alpha_{2}=\frac{k_{1}}{k_{2}}, \quad \alpha_{3}=\frac{E_{1}}{E_{2}}, \quad \beta=\frac{c_{p} E_{2}}{\left|Q_{2}\right| R} .
$$

For the unburnt region, $\xi \rightarrow-\infty$, we have $u=0, y_{1}=a$. For the burnt region, $\xi \rightarrow \infty$, we have $\frac{\partial u}{\partial \tau}=0, y_{1}=0$.

In general we may assume that $Q_{1}$ and $Q_{2}$ are not always positive. We note, however, that $Q_{1}$ and $Q_{2}$ can't both be negative, since in this case there will be no travelling wave in the combustion system. If $\alpha_{0}=1$ then the second reaction in (1) is exothermic, otherwise $\alpha_{0}=-1$ and the second reaction is endothermic. The parameter $\alpha_{1}$ presents the ratio of heat release of the first reaction to the second reaction. It is positive only if both reactions are exothermic and negative if the first reaction is endothermic. The parameter $\alpha_{2}$ is the ratio of the pre-exponential constants of two reactions and $\alpha_{3}$ is the ratio of their respective activation energies. The parameter $\beta$ expresses the ratio of activation energy needed by the second reaction to its energy release. 
Table 1: The sign of parameters under different situation

\begin{tabular}{ccc}
\hline & $\alpha_{0}$ & $\alpha_{1}$ \\
\hline Both reactions are exothermic & positive & positive \\
The relevant reaction of $y_{1}$ is endothermic & positive & negative \\
The relevant reaction of $y_{2}$ is endothermic & negative & positive
\end{tabular}

\section{NUMERICAL SOLUTIONS}

The governing equations (5) - (7) are analysed using a commercial finite element package (FlexPDE). It is a space- and time- adaptive finite element package which minimizes errors to a relative error tolerance level. If any step size exceeds this tolerance, the scheme refines the step size and the solution is recomputed. Solutions were also independently verified using Method of Lines (MOL). The key technique of the MOL is that the partial derivatives in spatial co-ordinates are replaced by finite difference approximators. In this way, each PDE is replaced by a system of ordinary differential equations (ODEs) in time allowing the application of standard ODE solutions. We have utilized MATLAB ${ }^{\mathrm{TM}}$ to solve the system of ODEs.

We have used the same parameter values considered by Please et al. (2003), namely $\alpha_{0}=-1, \alpha_{2}=2$ and $\alpha_{3}=1$, and throughout this investigation we have fixed the values of these parameters together with $\alpha_{1}=3.5$. The latter parameter represents the ratio of the heat released by the exothermic to the endothermic reactions. For values of $\alpha_{1}<3.5$, the travelling wave solution becomes unreliable as there were issues of convergence for the numerical schemes used. We hope to resolve these issues in future work.

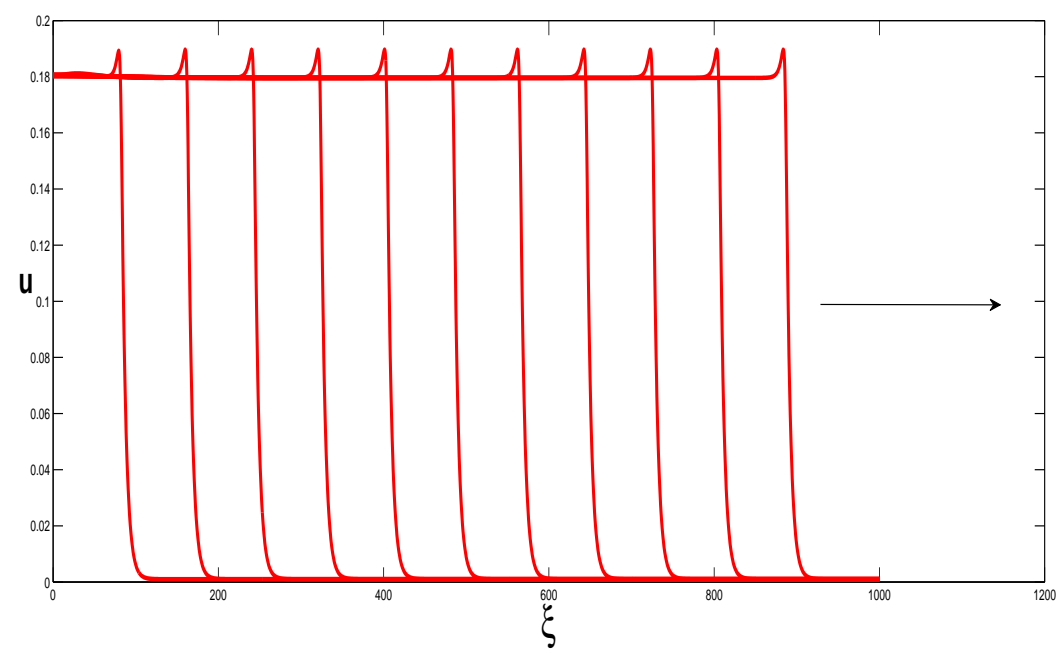

Figure 1: Structure of the temperature transient using PDE model for $\tau=400, \cdots, 4400\left(\alpha_{1}=3.5\right.$, $\beta=14)$.

Figure 1 shows the evolution of the temperature front and the profiles bear strong resemblance to the solutions obtained by Please et al. (2003) for $\alpha_{1}=5$. Here, the drop after the local maximum is larger as the effects of the endothermicity is greater for $\alpha_{1}=3.5$ than that for $\alpha_{1}=5$ used in Please et al. (2003). 


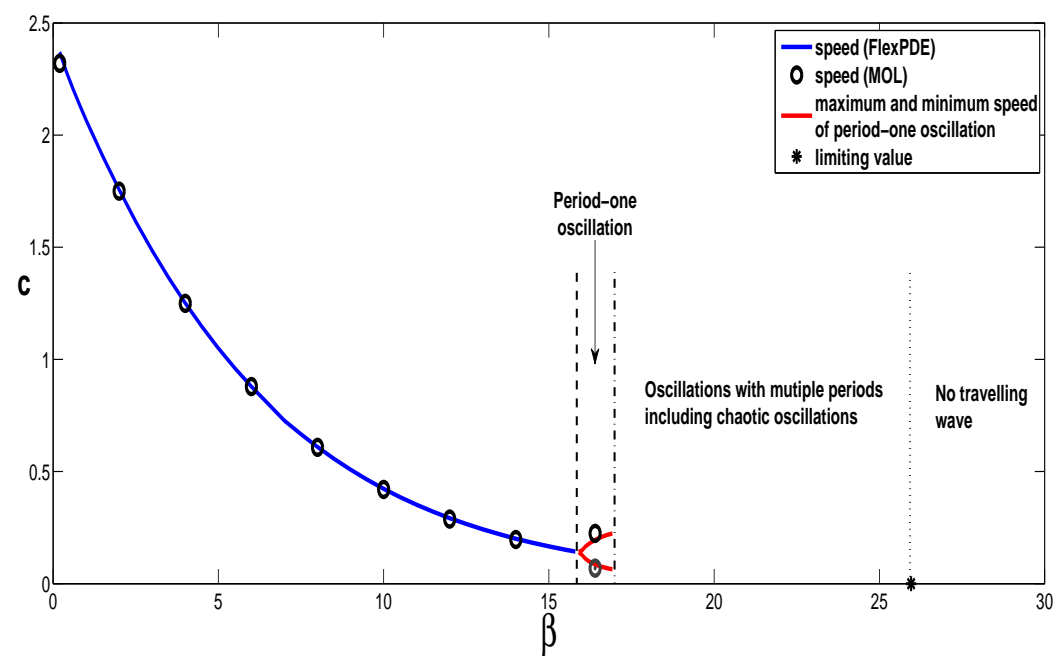

Figure 2: The dependence of speed upon $\beta$ for $\alpha_{1}=3.5$. The bold curves are solutions obtained using FlexPDE. The circles are solutions obtained by MOL. The red curves show the maximum and minimum values of the oscillation in the speed of the front.

Figure 2 illustrates the dependence of the combustion wave speed $c$ on the parameter $\beta$. In particular it shows how the speed varies over a wide range of $\beta$ values, not just the large $\beta$ treated by Please et al. (2003). The wave speed behavior is similar to that seen in the case of the single reactant model (Weber et al. (1997)). According to the parameter values used, both reactions have the same activation energies, and the heat released by the exothermic reaction is 3.5 times that absorbed by the endothermic reaction. This means that in the particular combustion system corresponding to these parameter values, the exothermic reaction dominates. At approximately $\beta=15.87$, the system appears to exhibit a Hopf bifurcation, beyond which oscillatory solutions are obtained. The two wave speed curves seen in Figure 2 for this region of parameter space indicate the minimum and maximum speeds of the pulsating solutions. The exact location of this suspected, Hopf bifurcation needs further investigation using the Evans function method (Sandstede (2002)). Furthermore, between $\beta=16.93$ and $\beta=25.98$, the system exhibits a sequence of oscillations with multiple periods. No solutions were able to be discerned using FlexPDE beyond $\beta=25.98$. Given the similarity of the wave speed behaviour described above to other combustion problems that display similar behaviour it is likely that the combustion waves become chaotic (through a period-doubling bifurcation) before reaching the extinction point. The oscillatory behaviour and the route to extinction requires closer examination and is a subject for future study.

The circles in figure 2 represents the speeds obtained using MOL. It is clear that the solutions obtained by MOL show a high level of correlation with those obtained using FlexPDE, particularly in the region with a single wave speed. However, for the region where pulsating wave exists (oscillatory behaviour), the error between the speeds obtained via MOL and FlexPDE are greater, with differences of maximum and minimum speed values being $15 \%$ and $19 \%$ respectively.

Figure 3 shows the variation in the speed of the combustion front over time corresponding to several values of $\beta$. Figure 3(a) shows that the wavespeed converges to a constant value at $\beta=14$. Figure 3(b) illustrates period-1 oscillations at $\beta=16.7$. The crest of wavespeed is $c=0.214$ and the trough of wavespeed is $c=0.071$. Figure 3(c) shows the development of a period- 2 oscillation at $\beta=18$.

\section{DiscuSSION AND CONCLUSIONS}

Please et al. (2003) established conditions in the limiting large activation energy limit for the three possible situation of the temperature profile in the whole process of travelling wave. This contribution is 


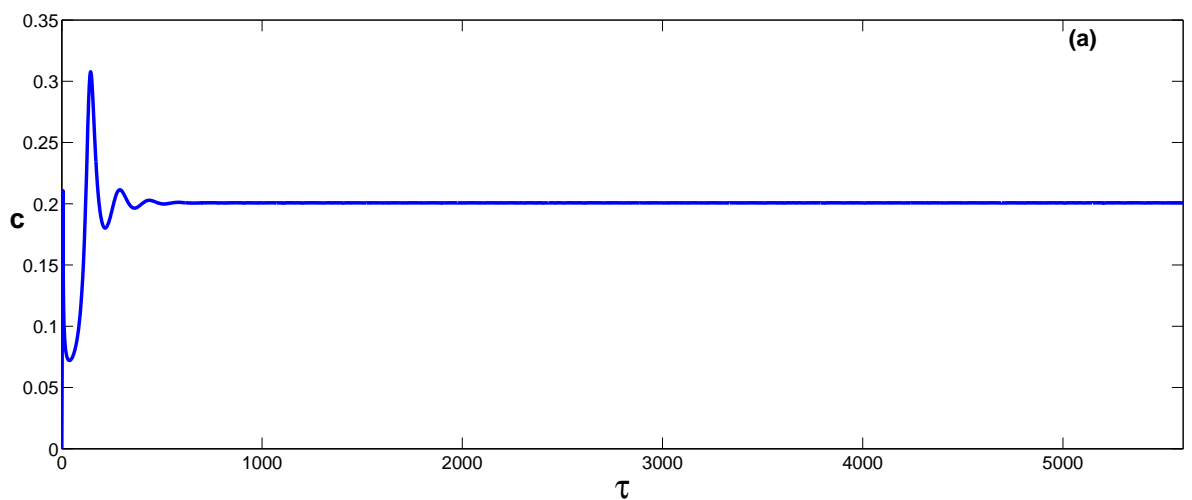

(a) Steady regime with the solution converging to a single speed.

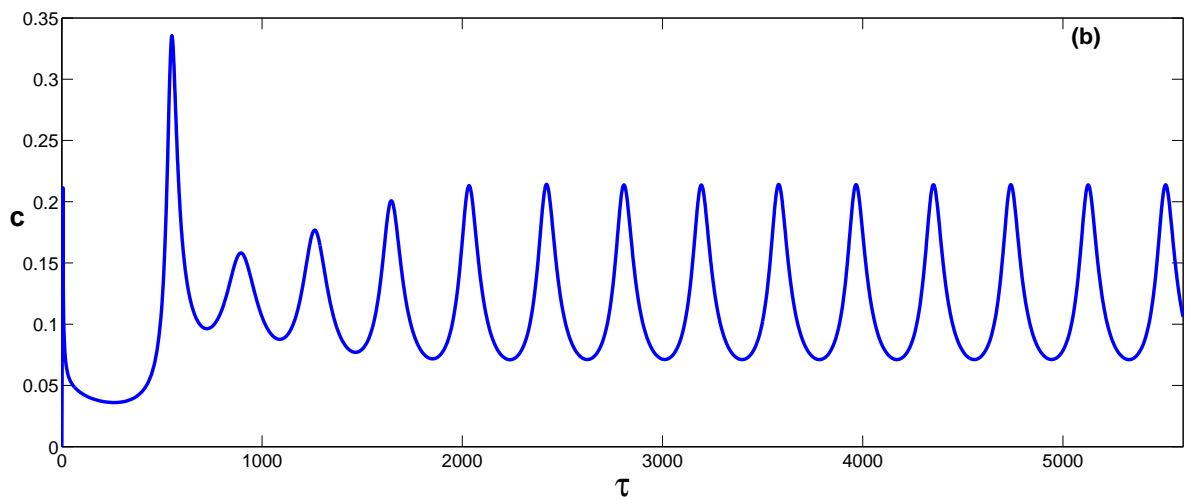

(b) Period-one oscillatory regime.

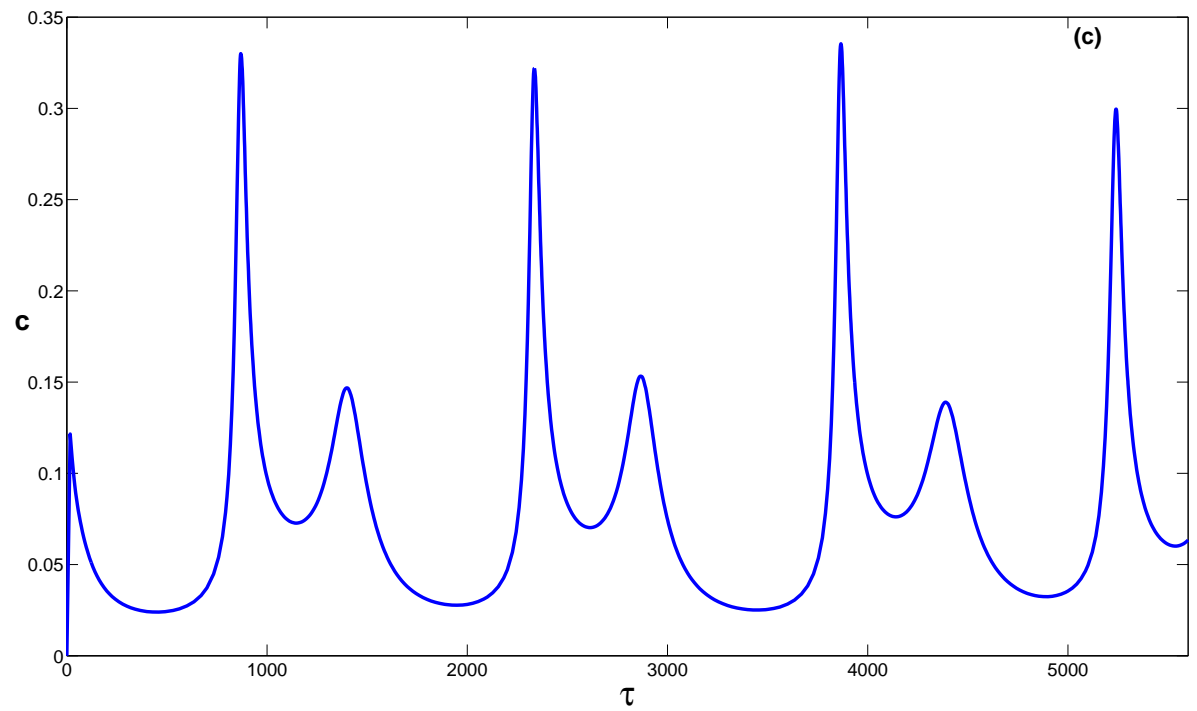

(c) Period-two oscillation.

Figure 3: The dependence of combustion wave speed upon time for $\alpha_{1}=3.5$. (a) convergence to a constant wave speed at $\beta=14$, (b) period- 1 oscillations at $\beta=16.7$ and (c) period-2 oscillation at $\beta=18$. 
a further study of the combustion system. By applying a finite element solver and the method of lines solving the governing equations, the travelling wave speed has been obtained numerically. The results presented here indicate that when $\beta$ increases beyond a certain limit solutions cannot be resolved using these numerical schemes - some differences were found between this limiting value of $\beta$ depending on the numerical scheme that was used. This limiting value was also mildly sensitive to the initial conditions, but this is expected given the nature of the numerical schemes.

It was also shown that for different values of the parameter $\alpha_{1}$, which describes the ratio of the heats of the endothermic and exothermic reactions, the characteristics of the oscillatory combustion waves varied. Smaller values of $\alpha_{1}$ resulted in oscillatory combustion wave behaviour for smaller values of $\beta$, i.e. the Hopf point values were seen to decrease with $\beta$. For $\alpha_{1}=3.5$, the Hopf point corresponded to $\beta=15.87$.

A lot of the analyses of combustion schemes and their solutions have focussed on high activation energy asymptotics (i.e. large $\beta$ ). Our results indicate that at large values of $\beta$ the behaviour of combustion waves can actually become quite complex, and so the results based on asymptotic analyses should be viewed with some caution.

Future work will continue to examine the behaviour of the sequential exothermic-endothermic combustion scheme for smaller values of $\alpha_{1}$. We will also extend the analyses by using more advanced numerical techniques and results will be compared and verified.

\section{ACKNOWLEDGEMENT}

The authors would like to acknowledge the financial support from the Australian Research Council Grant DP0878146.

\section{REFERENCES}

Dold, J. W. (2007). Premixed flames modelled with thermally sensitive intermediate branching kinetics. Combust.Theor. Model 11, 909-562.

FlexPDE $E^{T M}$. http://www.pdesolutions.com/.

Gubernov, V. V., H. S. Sidhu, and G. N. Mercer (2006). Combustion waves in a model with chain branching reaction. J. Math. Chem 39, 1-14.

Gubernov, V. V., H. S. Sidhu, G. N. Mercer, A. Kolobov, and A. Polezhaev (2008). The effect of Lewis number variation on combustion waves in a model with chain-branching reaction. J. Math. Chem 44, 816-830.

Hewitt, J. E. (1995). Proceedings of 1994 mathematics-in-industry study group. University of South Australia.

Hmaidi, A., A. C. Mclntosh, and J. Brindley (2010). A mathematical model of hotspot condensed phase ignition in the presence of a competitive endothermic reaction. Combustion Theory and Modelling 14, $893-920$.

Liu, F., D. L. S. McElwain, and C. P. Please (1998). Simulation of combustion waves for two-stage reactions. Computational Techniques and Applications, 385-392.

Matlab ${ }^{T M}$. Mathworks. http://www. mathworks.com/.

Mclntosh, A. C., R. O. Weber, and G. N. Mercer (2004). Non-adiabatic combustion waves for general Lewis numbers: Wave speed and extinction conditions. ANZIAM 46, 1105-118.

Please, C. P., F. Liu, and D. L. S. McElwain (2003). Condensed phase combustion travelling waves with sequential exothermic or endothermic reactions. Combustion Theory and Modelling 7:1, 129-143.

Sandstede, B. (2002). Stability of travelling waves. In Handbook of dynamical systems II (ed. B. Fiedler), 983-1055. Elsevier. 
Sharples, J. J., H. S. Sidhu, A. C. Mclntosh, J. Brindley, and V. V. Gubernov (2011). Analysis of combustion waves arising in the presence of a competitive endothermic reaction. IMA Journal of Applied Mathematics (under review).

Simon, P. V., S. K. Scott, S. Kalliadasis, and J. H. Merkin (2005). The effect of a radical scavenger on the propagation of flames in an exothermic-endothermic system. J. Math. Chem 38, 203-231.

Weber, R. O., G. N. Mercer, H. S. Sidhu, and B. F. Gray (1997). Combustion waves for gases $(L e=1)$ and solids $(L e \rightarrow \infty)$. Proceedings of The Royal Society A: Mathematical, Physical and Engineering Sciences 453, 1105-118. 